\title{
Determination of Sun Protection Factor (SPF) and Stability of Oil-in-Water Emulsions Containing Greek Red Saffron (Crocus Sativus L.) as a Main Antisolar Agent
}

\author{
Maria Zarkogianni*, Nikolaos Nikolaidis \\ Departement of Chemistry, Aristotle University of Thessaloniki, University Campus 54124, \\ Thessaloniki, Greece, \\ zarkogia28@yahoo.gr,nfnikola@chem.auth.gr
}

\begin{abstract}
The purpose of this study was to investigate the effects of Greek red saffron (Crocus Sativus L.) as a component in cosmetic sunscreen products. For the preparation of oil-in-water emulsions saffron used either as an aqueous solution $(1 \% \mathrm{w} / \mathrm{v}$ and $2 \% \mathrm{w} / \mathrm{v})$ or as ground stigmas $(1 \% \mathrm{w} / \mathrm{w}$ and $2 \% \mathrm{w} / \mathrm{w})$. The pollens of the saffron were dried and powdered in a grinder. A control reference emulsion without saffron was also prepared. The sun protection factors (SPFs) of the emulsions were determined by anin vitroultraviolet spectrophotometry method. The stability of emulsions during time were evaluated by measuring $p H$, conductivity and viscosity of the emulsions. It was concluded from this study that the SPF of saffron emulsions in all cases were significantly higher than that of the reference emulsion and that as the proportion of saffron increases the SPF values increases too. The emulsions containing the ground stigmas give a slightly higher SPF values than that of the emulsions containing aqueous solutions of saffron. These results show that saffron can act as a very good antisolar agent against the harmful UV radiation.
\end{abstract}

Keywords: Saffron; Antisoloar; Sun protection factors; Emulsions Stability

\section{INTRODUCTION}

In the last few years, in the field of cosmetology, physical products have started to gain the interest of consumers. Because of the constantly growing use of chemicals and their harmful consequences in peoples' health, scientists started to look for alternative findings to surpass these problems. Thus herbals have gain attention in the cosmetology [1-4].

Eco-friendly cosmetics appeared in the early 1900s. The biggest advantage of these cosmetics is that they are safe for humans and the environment as they do not contain any toxic chemicals, they need no animal testing and their results can be as pleasing as those achieved by commercial products [5].

Nowadays the rapid growth of sunscreens' marketing indicates that even though a suntan is still desired, people are nevertheless quite conscious of accompanying dangers like wrinkling, premature ageing of the skin, irregular thinning of the epidermis, solar keratosis and skin cancer occurring as a result of excessive exposure to ultraviolet (UV) radiation [6, 7]. Every year, more than one million people are diagnosed with skin cancer and about 10,000 die from malignant melanoma [8].

The harmful effects of solar radiation are caused predominantly by the ultraviolet (UV) region of the electromagnetic spectrum, which can be divided into three regions: UVA (320 to $400 \mathrm{~nm}$ ), UVB (290 to $320 \mathrm{~nm}$ ) and UVC ( 200 to $290 \mathrm{~nm}$ ). UVC radiation is filtered by the atmosphere before reaching earth. UVB radiation is not completely filtered out by the ozone layer and is responsible for the damage due to sunburn. UVA radiation reaches the deeper layers of the epidermis and dermis and provokes the premature ageing of the skin [9].

The efficacy of a sunscreen is usually expressed by the sun protection factor (SPF), which is defined, as the UV energy required to produce a minimal erythema dose (MED) on protected skin, divided by the UV energy required to produce a MED on unprotected skin. The minimal erythemal dose (MED) is defined as the lowest time interval or dosage of UV light irradiation sufficient to produce a minimal, perceptible erythema on unprotected skin [10]. The higher the SPF, the more effective is the product in preventing sunburn. 


$$
S P F=\frac{\text { Minimal erythema dose in sunscreen }- \text { protected skin }}{\text { Minimal erythema dose in nonsuscreen }- \text { protected skin }}
$$

Nevertheless, it is necessary to standardize methods to determine the SPF of commercially available products. The most traditional and officially accepted method in several countries is the in vivo method of determination of SPF (FDA, United States; DIN, Germany; COLIPA, European Union; AAN, Australia). All of these are long range methods and involve 10 to 20 human volunteers of both sexes, with appropriate skin types. The in vivo method is expensive and introduces the ethical consideration of human testing [11]. As a result of this, scientists were putting efforts to develop an in-vitro technique in assessing the efficiency of sunscreen products.

The in-vitro approaches are generally two types. 1) Measurement of absorption or the transmission of UV radiation through sunscreen product films in quartz plates or membranes 2) methods in which the absorption characteristics of the sunscreens agents are determined based on spectrophotometric analysis [12]. Mansur et al. developed a very simple mathematical equation to estimate the sun protection factor by in-vitro method using UV spectrophotometry [13]. The major advantage of the in vitro test is that it is a rapid, objective, cost-effective screening methodology. In vitro testing can be used as a formulation tool to identify new filters, optimize combinations of old ones, and prescreen protective formulas prior to in vivo testing in humans [14, 15].

Natural substances extracted from plants have recently been considered as potential sunscreen resources because of their ultraviolet absorption in the UV region and their antioxidant activity [1621].

Saffron is the aqueous extract of the dried stigmas of a flower scientifically identified as Crocus Sativus L. Saffron was known by ancient nations and has remained among the world's costliest substances throughout the history [22]. It is native to Iran and Greece. It is now cultivated largely in Southern Europe, Tibet and other countries. In India it is mainly cultivated in Kashmir and Uttranchal. It is widely used as spice and as a coloring and flavoring agent in the preparation of various foods and cosmetics. The stigmas of the plant are mainly used for therapeutic purposes. Topically it is applied in the form of paste to treat skin diseases like acne [23].

The chemical composition of stigmas of Crocus Sativus L. has been investigated in several studies during the past two decades. Stigmasof Crocus Sativus flower contain three main metabolites; 1) Crocins which are the saffron-colored compounds (unusual water-soluble carotenoids due to their high glycosyl contents) 2) Picrocrocins which are the main substances responsible for saffron's bitter taste and 3) Safranal which is the volatile oil responsible for the typical saffron aroma. In addition to crocin and picrocrocin, the major compounds in saffron, anthocyanins, flavonoids, vitamins, amino acids, proteins, starch, mineral matter, gums and other chemical compounds have been found in saffron. Figure 1 illustrates the structure of the most important component of saffron [24, 25].
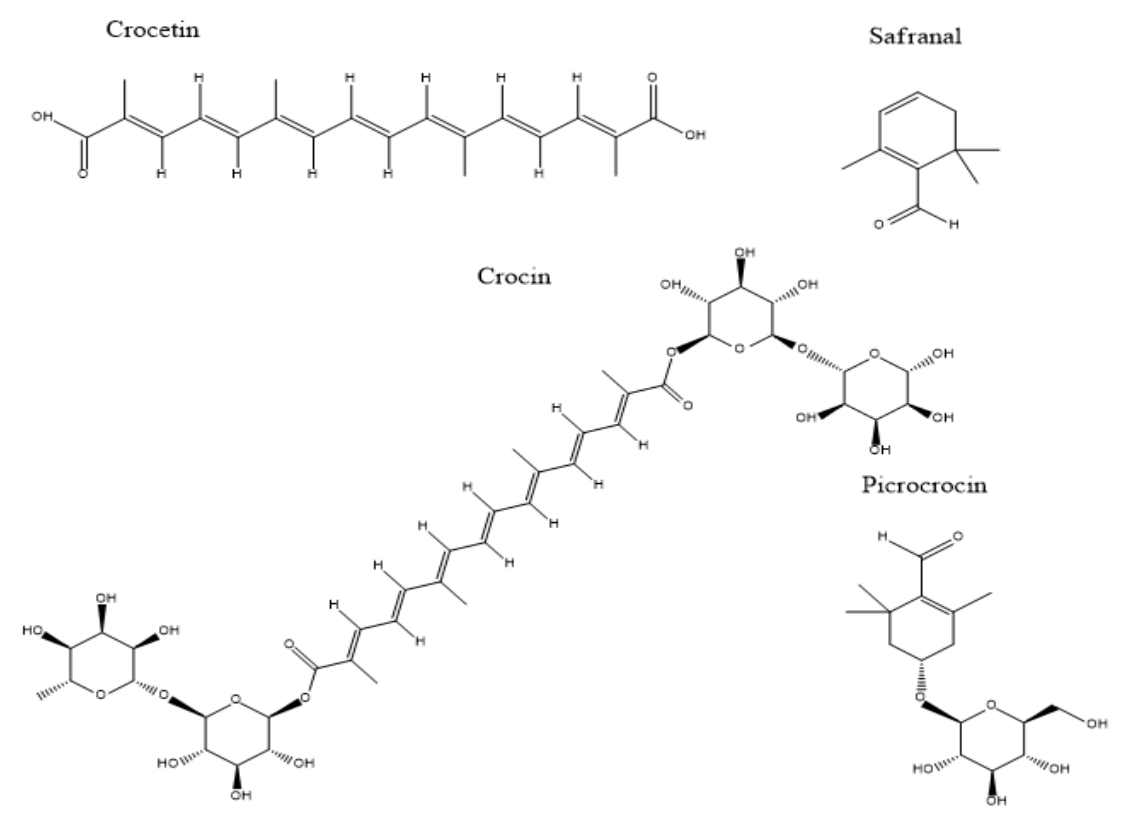

Figure1. Chemical composition of the most active constituents of saffron 
A series of oil-in-water emulsions was prepared with different amount either of aqueous extracts of saffron or grained stigmas. Olive oil was used for the preparation of the oily phase of the emulsion. The selected emulsifier was a combination of polysorbate 60 (polyoxythylene sorbitan monostearate) and cetearyl alcohol. The SPF values of the oil-in-water emulsions containing saffron was determined by an in vitro method. Additionally $\mathrm{pH}$, surface tension, conductivity and viscosity measurements were made to the emulsion during time. Evaluation of the results are presented and discussed in detail.

\section{MATerial AND MethodS}

\subsection{Materials}

A commercial sample of saffron stigmas (Cooperative de Safron, Crocos, Kozani, Greece) was used. The sample was a representative mixture of saffron stigmas dried in air in the air (dark) from the areas in Northern Greece where saffron is exclusively cultivated. Tetrasodium EDTA, xanthan gum, cetearyl alcohol and polysorbate 60 were purchased from NOVITA Group (MariseParfumes). Glycerin was obtained from Emery Oleochemicals. Cetyl alcohol was obtained from BASF. Stearic acid was purchased from NAPOLEO (Natural Oleochemicals). Caprylic/Capric triglyceride was obtained from CRODA. ButyrospermumParkii (Shea) Butter was obtained from A. \& A. FratelliParadis.p.a. and 2-phenoxyethanol from Sharon Laboratories. Greek extra virgin Olive oil was obtained by "MINERVA" and ethanol (pro analysis) from MERCK.The above compounds were used to the preparation of the emulsions (Table1).

\subsection{Apparatus}

A Smimadzu UV-2101 spectrophotometer equipped with $1 \mathrm{~cm}$ quartz cell was used. $\mathrm{pH}$ measurements were made using a WTW pH535 microprocessor. Surface Tension measurements were taken with the Wilhelmy plate technique using a KSV Sigma 70 tensionmeter. The viscosity values were taken by using the viscometer Visco Star Plus. The overhand mixer used was obtained from IKA-WERCK (RW 14H). The ultrasonic processor that was used was UP 100H from Hielscher and the conductivity measurements were taken by a KRISON Basic 30 conductivity meter.

\subsection{Extraction of Saffron}

The stigmas of saffron (Crocus Sativus L.) were placed into a beaker with de-ionised water in a proportion of $2 \% \mathrm{w} / \mathrm{v}$. The material was warmed up for almost 3 hours at $80-90{ }^{\circ} \mathrm{C}$ with simultaneous stirring and then was kept until next morning in room temperature and in dark in order to have a complete extraction. The aqueous phase was selected and dried until the dry ground saffron was obtained.

\subsection{Emulsification Process}

Emulsification was implemented by mixing the water phase with the oily phase (Table 1) using an impeller rotating at the central axis of a glass vessel. Emulsions were prepared in the above glass vessel as follows. Initially the aqueous phase was heated to $70^{\circ} \mathrm{C}$. The appropriate amount of the oily phase was heated separately to $70^{\circ} \mathrm{C}$ until the material was melt. Emulsification started by adding the hot oily phase into the aqueous phase under stirring. At that moment heat supply was turned off and agitation was set at 700rpm and continued for almost 2 hours until the emulsion to cool down to room temperature. Phenoxyethanol was added at the emulsion at about $50{ }^{\circ} \mathrm{C}$. In Table 1 the emulsion constituents with their \% content are given.

Table1. Emulsion constituents with their \% content

\begin{tabular}{|l|l|}
\hline Aqueous Phase (70\%) & \multicolumn{2}{|l|}{} \\
\hline Constituents & $67 \%$ \\
\hline Water or Aqueous extract of Saffron & $2.3 \%$ \\
\hline Glycerin & $0.2 \%$ \\
\hline Tetrasodium EDTA & $0.5 \%$ \\
\hline Xanthan Gum & \\
\hline Oily Phase (30\%) & $13 \%$ \\
\hline Constituents & $4 \%$ \\
\hline Olive Oil & $2 \%$ \\
\hline Polysorbate 60+Cetearyl Alcohol (1:1) & \\
\hline Cetyl Alcohol & \\
\hline
\end{tabular}




\begin{tabular}{|l|l|}
\hline \hline Stearic Acid & $1 \%$ \\
\hline Triglyceride & $7 \%$ \\
\hline Shea Butter & $2 \%$ \\
\hline Phenoxyethanol & $1 \%$ \\
\hline
\end{tabular}

\subsection{Sample Preparation and Determination of SPF}

All samples $(1 \mathrm{~g})$ were weighed, transferred to a $100 \mathrm{~mL}$ volumetric flask, diluted to volume with ethanol, mixed for $15 \mathrm{~min}$ and then filtered through Whatman filters. A $5.0 \mathrm{~mL}$ sample was transferred to a $25 \mathrm{~mL}$ volumetric flask and diluted to volume with ethanol. The absorption values were obtained in the range of 290 to $320 \mathrm{~nm}$ (every $5 \mathrm{~nm}$ ). Each measurement was conducted three times and the value given is the mean value at each point. Then, Mansur equation was used to determine the SPF values of the formulations. Mansur et al. (1986) developed a very simple mathematical equation, which in conjunction with substitutes the in vitro method proposed by Sayre et al. (1979), UV spectrophotometry. The introduced equation is as follows:

$S P F=C F \times \sum_{320}^{290} \quad \mathrm{EE}_{(\lambda)} \cdot \mathrm{I}_{(\lambda) \cdot} \cdot \operatorname{abs}_{(\lambda)}$

where $\mathrm{CF}=10$ (Correction Factor), $\mathrm{EE}_{(\lambda)}=$ Erythemogenic Effect of radiation at wavelength $\lambda$, I ${ }_{(\lambda)}=$ Intensity of solar light at wavelength $\lambda$, and $\mathrm{abs}_{(\lambda)}=$ Absorbance of wavelength $\lambda$ by a solution of the preparation. It was determined so that a standard sunscreen formulation containing $8 \%$ homosalate presented an SPF value of 4 , determined by UV spectrophotometry [32, 33]. The values for the term "EE x I" are constants, which were determined by Sayre et al. (1979), and are shown in Table 2 [34].

Table2. Normalized product function used in the calculation of SPF

\begin{tabular}{|l|l|}
\hline Wavelength $(\lambda \mathrm{nm})$ & EE x I (normalized) \\
\hline 290 & 0.0150 \\
\hline 295 & 0.0817 \\
\hline 300 & 0.2874 \\
\hline 305 & 0.3278 \\
\hline 310 & 0.1864 \\
\hline 315 & 0.0839 \\
\hline 320 & 0.0180 \\
\hline Total & 1 \\
\hline
\end{tabular}

\section{6.pH, Conductivity, Viscosity and Surface Tension Measurements}

$\mathrm{pH}$ and conductivity measurements were made by dipping the $\mathrm{pH}$ or the conductivity sensor into the emulsion. Viscosity measurements were made by dipping the spindle R3 of the viscometer into the emulsion. The surface tension measurements were taken with the Wilhelmy plate technique. The platinum plate was partially immersed into the oil or the aqueous phase, where it remained to fixed position during the course of the experiment. Thus, the surface tension decreased as a function of time. When the surface tension was stabilized then the experiment was stopped. All measurements were made at $25{ }^{\circ} \mathrm{C} \pm 0.1{ }^{\circ} \mathrm{C}$.

\section{RESUlts AND DisCuSSION}

\subsection{SPF Determination}

In this study all emulsions with and without saffron were evaluated by UV spectrophotometry using Mansur mathematical equation. The SPF values of the emulsions with $0.05 \% \mathrm{w} / \mathrm{v}$ and $0.1 \% \mathrm{w} / \mathrm{v}$ aqueous solution of saffron (Emulsion I and II respectively), emulsions with ground stigmas $1 \% \mathrm{w} / \mathrm{w}$ and 2\%w/w (III and IV respectively) and the reference emulsion containing no saffron (R) were measured. Figure 2 shows the SPFs of emulsions. It was concluded from this that the SPF of saffron emulsion in all cases were significantly higher than that of the referenceemulsion and that as the proportion of saffron increases the SPF values increases too. The emulsions containing the ground stigmas give a slightly higher SPF values than that of the emulsions containing aqueous solutions of saffron. These results show that saffron can act as a very good antisolar agent against the harmful UV radiation. 


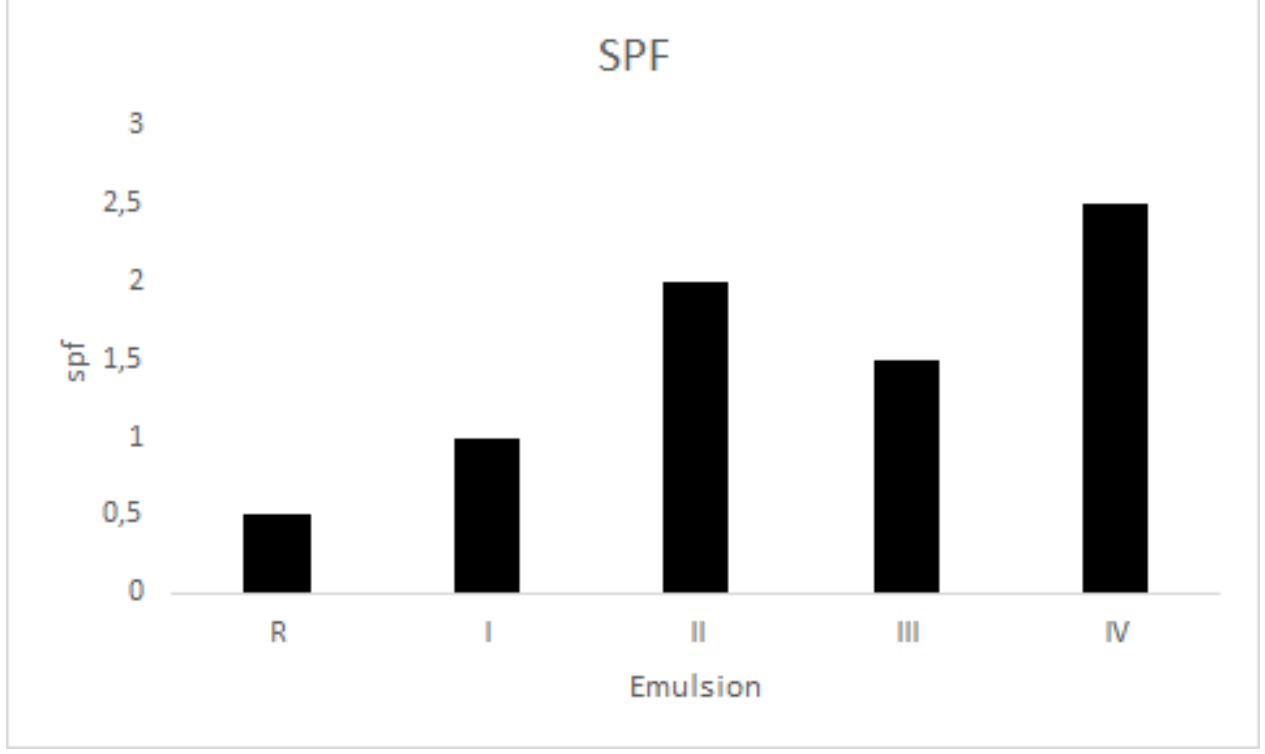

Figure2. $S P F$ values of the emulsions

\section{2.pH, Conductivity, Viscosity and Surface Tension Measurements}

The measurements of $\mathrm{pH}$, conductivity and viscosity of the emulsions during time are shown in Figure 3. All the emulsions show remarkable $\mathrm{pH}$ and viscosity stability during time. The conductivity in all cases shows slight decrease during time. The surface tension values of the aqueous and oily phases are shown in Table 3 and are in accordance with the literature data.
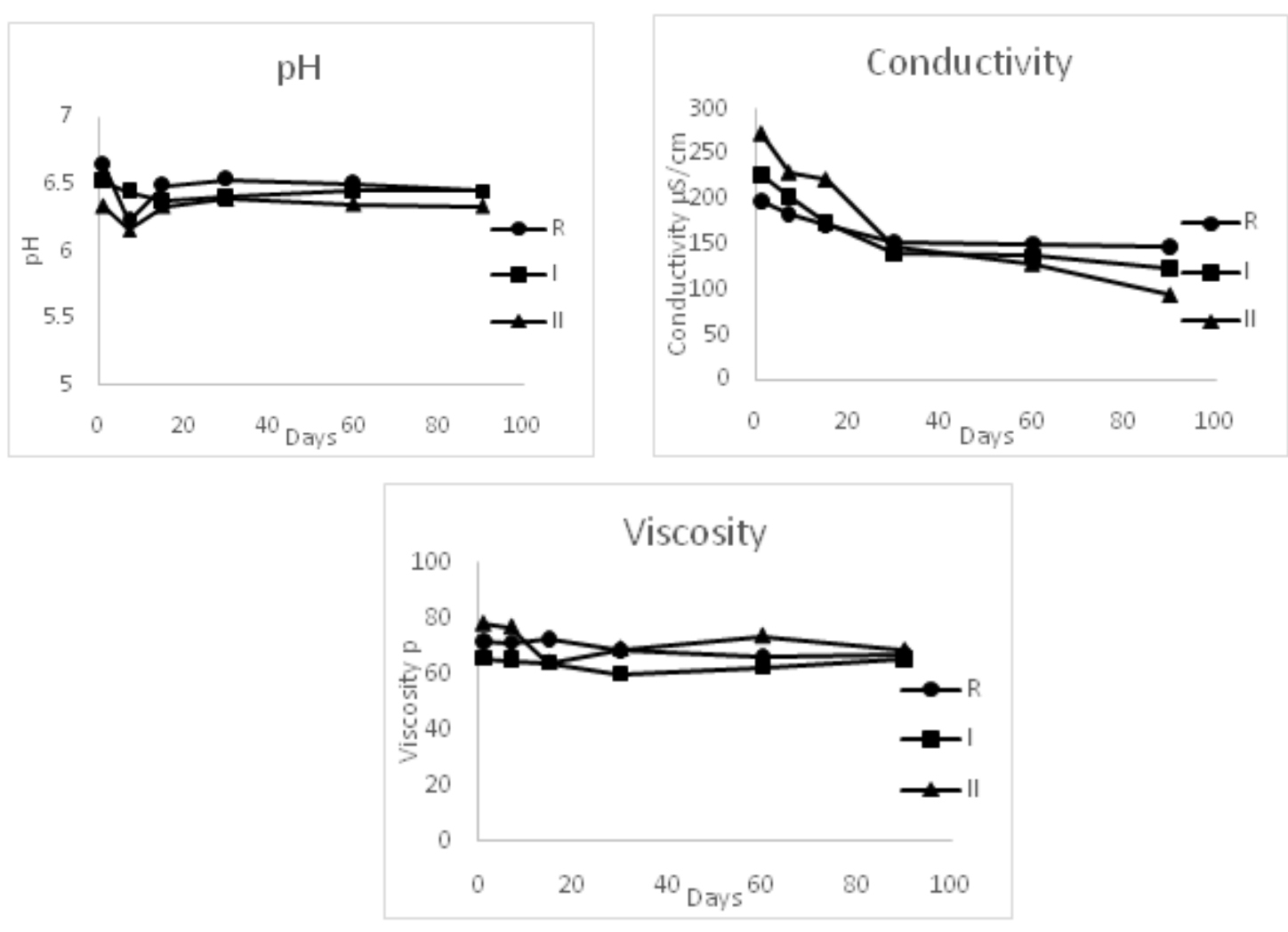

Figure3. $p H$, Conductivity and Viscosity measurements of the emulsions during time

Table3. Surface tensions of oily and aqueous phases

\begin{tabular}{|l|l|l|}
\hline Phase & Temperature $\left({ }^{\circ} \mathrm{C}\right)$ & Surface tension $(\mathrm{mN} / \mathrm{m})$ \\
\hline Olive oil & $25 \pm 0.1$ & 31 \\
\hline Water & $25 \pm 0.1$ & 71.6 \\
\hline Aqueous solution 0.1\% saffron & $25 \pm 0.1$ & 48.6 \\
\hline Aqueous solution 0.05\% saffron & $25 \pm 0.1$ & 50.9 \\
\hline
\end{tabular}




\section{Conclusion}

The results of this present work indicated that saffron can be used as a natural UV-absorbing agent in sunscreen cosmetic products and that as the proportion of saffron increases the SPF values increases too. The emulsions containing the ground stigmas give a slightly higher SPF values than that of the emulsions containing aqueous solutions of saffron. Viscosity, $\mathrm{pH}$ and conductivity measurements indicated that the emulsions made in all cases are significant stable during time. The above results in combination with the all the significant properties of saffron known from the literature are very promising in the direction of an additional use of saffron in cosmetics and especially in sunscreen products.

\section{ACKNOWLEDGEMENTS}

This study was financially supported by "IKY FELLOWSHIPS OF EXCELLENCE FOR POSTGRADUATE STUDIES IN GREECE-SIEMENS PROGRAM".

\section{REFERENCES}

Aburjai T., NatshehFM.. Plants used in cosmetics. Phytotherapy Research.17, 987-1000(2003).

Wang KH., Lin RD., Hsu FL., Huang YH., Chang HC., Huang CY., Lee MH..Cosmetic applications of selected traditional Chinese herbal medicines. Journal of Ethnopharmacology. 106(3), 353359(2006).

Smith H.R., Armstrong D.K.B., Holloway D., Whittam L., Basketter D.A., Mcfadden J.P..Skin irritation thresholds in hairdressers: implications for the development of hand dermatitis. British Journal of Dermatology. 146(5), 849-852(2002).

Regnier M., Duval C., Schmidt R..Potential cosmetic applications of reconstructed epidermis.Int J Cosmet Sci. 21(1), 51-58(1999).

Varka E-M., Tsatsaroni E., Xristoforidou N., Darda A-M..Stability Study of O/W Cosmetic Emulsions Using Rosmarinus officinalis and Calendula officinalis Extracts.Open Journal of Applied Sciences. 2, 139-145(2012).

Gaspar LR., Maia Campos PM..Rheological behavior and the SPF of sunscreens.Int J Pharm.250(1), 35-44(2002).

Pathak M.A., Fitzpatrick T.B., Greiter F., KrausE.W..Preventive treatment of sunburn, dermatoheliosis, and skin cancer with sun-protective agents.Dermatology in general medicine. 4, 1689-717(1993).

Golmohammadzadeh S., Jaafari MR., Hosseinzadeh H..Does saffron have antisolar and moisturizing effects? Iran J Pharm Res. 9(2), 133-40 (2010).

Dutra E-A., Oliveira D-A., KedorHackmann E-R., Santoro M.I.. Determination of sun protection factor (SPF) of sunscreens by ultraviolet spectrophotometry. Brazilian Journal of Pharmaceutical Sciences. 40(3), 381-385(2004).

Wood C., Murphy E..Sunscreens efficacy. Glob. Cosmet. Ind. 167, 38-44(2000).

Santos E.P., Freitas Z.M., Souuza K.R., Gracia S., VergnaniniA..In vitro and in vivo determinations of sun protection factors of sunscreen lotions with octylmethoxycinnamate.Int. J. Cosmet. Sci.21(1), 1-5 (1999).

Walters C., Keeney A., Wigal C.T., Johnstom C.R., Cornelius R.D.. UV spectra and cost analysis of suntan lotions: A simple introduction to the use of recording spectrophotometers. J. Chem. Educ. 74, 99-101(1997).

Mansur J.S., Breder M.N.R., Mansur M.C.A., AzulayR.D..Determinação Do Fator De Proteção Solar PorEspectrofotometria. An Bras Dermatol Rio De Janeiro. 61, 121-124(1986).

Mbanga L., Mulenga M., Mpiana P.T., Bokolo K., Mumbwa M., MvinguK.. Determination of Sun Protection Factor (SPF) of Some Body Creams and Lotions Marketed in Kinshasa by Ultraviolet Spectrophotometry. International Journal of Advanced Research in Chemical Science. 1(8), 7-13 (2014).

Sudhahar V., BalasubramanianV.. Sun production factor (SPF) determination of marketed sunscreen formulation by In-Vitro method using UV-VIS spectrophotometer. Archives of Applied Science Research, vol. 5(6), 119-122(2013). 
Determination of Sun Protection Factor (SPF) and Stability of Oil-in-Water Emulsions Containing Greek Red Saffron (Crocus Sativus L.) as a Main Antisolar Agent

Tabrizi S., Mortazavi S.A., Kamalinejad M.. An in vitro evaluation of various Rosa damascena flower extracts as a natural antisolaragent. Int. J. Cos. Sci.25, 259-265 (2003).

UrbachF.. The historical aspects of sunscreens. J. Photochem. Photobiol. B. 64, 99-104(2001).

Elmets C.A., Young C.. Sunscreens and photocarcinogenesis: An objective assessment. Photochem. Photobiol. 63, 435-439(1996).

Wang Z.Y., Agarwal R., Bickers D.R., Mukhtar H.. Protection against ultraviolet B radiation-induced photocarcinogenesis in hairless mice by green tea polyphenols. Carcinogenesis. 12, 15271532(1991).

Rancan F., Rosand S., Boehm K., Fernandez E., Hidalgo M.E., Quihot W.. Protection against UVB irradiation by natural filters extracted from lichens. J. Photochem. Photobiol. B. 68, 133139(2002).

Khazaeli P., Mehrabani M.. Screening of Sun Protective Activity of the Ethyl Acetate Extracts of Some Medicinal Plants. Iran. J. Pharmaceut.7, 5-9(2008).

Mousavi S.Z., Bathaie S.Z..Historical uses of saffron: Identifying potential new avenues for modern research. Avicenna Journal of Phytomedicine. 1(2), 57-66 (2011).

Bhargava V.K.. Medicinal uses and pharmacological properties of Crocus Sativus Linn (Saffron). International Journal of Pharmacy and Pharmaceutical Sciences. 3(3), 21-26(2011).

Mashmoul M., Azlan A., Khaza'ai H., Yusof B.N.M., Noor S.M..Saffron: A Natural Potent Antioxidant as a Promising Anti-Obesity Drug.Antioxidants. 2(4), 293-308(2013).

Tsatsaroni E.G., EleftheriadisI.C.. The colourand fastness of natural saffron.Journal of the Society of Dyers and Colourists. 110(9), 313-315(1994).

Sayre R.M., Agin P.P., Levee G.I., Marlowe E.. Comparison of in vivo and in vitro testing of sunscreening formulas. Photochem. Photobiol. 559-566(1979). 